\title{
Pseudosarcomatous fibroblasticl myofibroblastic proliferation in perinephric adipose tissue adjacent to renal cell carcinoma: a lesion mimicking well-differentiated liposarcoma
}

\author{
Munir R Tanas ${ }^{1}$, Chalenpoj Sthapanachai ${ }^{2}$, Daisuke Nonaka ${ }^{3}$, Jonathan Melamed ${ }^{3}$, \\ Andre M Oliveira ${ }^{4}$, Michele R Erickson-Johnson ${ }^{4}$ and Brian P Rubin ${ }^{1,5}$ \\ ${ }^{1}$ Department of Anatomic Pathology, Pathology and Laboratory Medicine Institute, The Cleveland Clinic and \\ The Cleveland Clinic Lerner College of Medicine, Case Western Reserve University, Cleveland, OH, USA; \\ ${ }^{2}$ Department of Anatomic Pathology, University of Washington Medical Center, Seattle, WA, USA; \\ ${ }^{3}$ Department of Pathology, New York University Medical Center, New York, NY, USA; ${ }^{4}$ Department of \\ Pathology, Mayo Clinic, Rochester, MN, USA and ${ }^{5}$ Department of Molecular Genetics, Lerner Research \\ Institute and Taussig Cancer Center, Cleveland, OH, USA
}

\begin{abstract}
Cytologically atypical stromal cells were found in the perinephric adipose tissue, mimicking well-differentiated liposarcoma in 12 of $59(20 \%)$ consecutive nephrectomy specimens that were resected for renal cell carcinoma. Morphologically, the atypical cells included enlarged, hyperchromatic spindle cells and floret-type multinucleate cells. Of $59,10(17 \%)$ renal cell carcinomas invaded through the renal capsule into the perinephric adipose tissue. Of these cases, three $(30 \%)$ contained the aforementioned atypical cells. In contrast, 9 of 49 cases without extrarenal invasion (18\%) contained the atypical stromal cells. Of the 12 cases with atypical stromal cells, $3(25 \%)$ were associated with extrarenal involvement. The atypical spindle cells exhibited focal to variable positivity for smooth muscle actin and desmin in 3 of the $\mathbf{1 4}$ cases (including two cases from our consultation files) each. Cytokeratin AE1/AE3, cytokeratin Cam 5.2, cytokeratin 7, epithelial membrane antigen, and S-100 were negative in all cases. Amplification of MDM2 gene region, which is commonly observed in welldifferentiated liposarcoma, was absent by fluorescence in situ hybridization (FISH) in the atypical stromal cells. Immunohistochemistry and FISH suggest that the atypical cells are most consistent with reactive fibroblasts/ myofibroblasts. Recognition of these atypical fibroblasts/myofibroblasts may help in avoiding the potential pitfall of misdiagnosing them as well-differentiated liposarcoma.
\end{abstract}

Modern Pathology (2009) 22, 1196-1200; doi:10.1038/modpathol.2009.84; published online 12 June 2009

Keywords: renal cell carcinoma; well-differentiated liposarcoma; MDM2; pseudosarcomatous fibroblastic/ myofibroblastic proliferation

There are approximately 36000 new cases of renal cell carcinoma each year. ${ }^{1}$ Four main histological subtypes have been described including clear cell, papillary, chromophobe, and collecting duct carcinomas. ${ }^{1}$ All four histological subtypes may be associated with a metaplastic spindle-cell compo-

Correspondence: Dr BP Rubin, MD, PhD, Department of Anatomic Pathology, Cleveland Clinic, 9500 Euclid Avenue, Cleveland, $\mathrm{OH}$ 44195, USA.

E-mail: rubinb2@ccf.org

Received 5 March 2009; revised 7 May 2009; accepted 8 May 2009; published online 12 June 2009 nent, often given the term 'sarcomatoid renal cell carcinoma,' which confers a poor prognosis. ${ }^{2,3}$ The histological appearance of metaplastic renal cell carcinoma is usually characterized by anaplastic appearing spindle cells. Heterologous differentiation has also been observed. ${ }^{2-4}$

Liposarcoma is the most common soft tissue sarcoma and accounts for at least $20 \%$ of all adulthood sarcomas. ${ }^{5}$ Three main histological subtypes have been described: well differentiated/ dedifferentiated, myxoid, and pleomorphic. ${ }^{5,6}$ Well-differentiated liposarcoma, also known as atypical lipomatous tumor, is the most common 
type, and reaches a peak incidence in the sixth and seventh decades of life. Dedifferentiated liposarcoma arises from well-differentiated liposarcoma and is essentially the high-grade form of well-differentiated liposarcoma., ${ }^{5,6}$ It can occur de novo or on recurrence of well-differentiated liposarcoma. One of the most common anatomic sites for welldifferentiated liposarcoma is the retroperitoneum. Well-differentiated liposarcoma, practically speaking, is a non-metastasizing sarcoma, however in the retroperitoneum, wide local excision is next to impossible and the recurrence rate approaches $100 \% .{ }^{6}$ Dedifferentiated liposarcoma has significant metastatic potential and an overall more aggressive clinical course than well-differentiated liposarcoma. With each additional recurrence, there is an increased risk of progression to dedifferentiated liposarcoma. ${ }^{5,6}$

Well-differentiated liposarcoma is characterized by two major subtypes, the adipocytic (lipoma-like) form, and the sclerosing form, which is found most commonly in the retroperitoneum. ${ }^{5,6}$ Both types contain well-differentiated adipocytes, which typically vary in size and shape and usually have broad fibrous bands/septa containing atypical spindle cells with hyperchromatic nuclei. Well-differentiated liposarcoma may also contain lipoblasts, however they are not always present and are not required for the diagnosis of well-differentiated liposarcoma. Lipoblasts are thought to represent immature adipocytes and characteristically, contain multiple lipid-filled cytoplasmic vacuoles that indent or 'scallop' a hyperchromatic nucleus.

Well-differentiated liposarcoma is characterized cytogenetically by the presence of ring (marker) chromosomes derived from large amplicons of chromosome $12 q 12-15 .^{7-9}$ The gene for MDM2, an inhibitor of TP53 has been localized to this amplicon and is implicated in the pathogenesis of well-differentiated liposarcoma. ${ }^{7-9}$ A method has been developed using fluorescence in situ hybridization (FISH) to evaluate for MDM2 gene region amplification in paraffin-embedded sections. ${ }^{10}$

We came across two cases of renal cell carcinoma in consultation with marked/bizarre cytological atypia in the perinephric fibroadipose tissue but no recognizable soft tissue mass, with the question posed of whether they were retroperitoneal welldifferentiated liposarcoma. This prompted us to examine additional cases to determine how common these changes are. We identified these findings in 12 $(20 \%)$ of 59 consecutive nephrectomy specimens resected for renal cell carcinoma.

\section{Materials and methods}

The clinical and pathological records of 59 consecutive nephrectomy specimens containing renal cell carcinoma, spanning 14 months in the University of Washington Medical Center surgical pathology files, were reviewed. Immunohistochemistry was performed by the avidin-biotinperoxidase complex using commercially available antibodies to the following antigens: pan-cytokeratin (AE1/AE3, 1:100; Dako, Carpinteria, CA, USA), Cam 5.2 (1:50; BD Biosciences Transduction Lab, Franklin Lakes, NJ, USA), cytokeratin 7 (OV-TL, 1:500; Dako), EMA (E29, 1:1000; Dako), S-100 protein (polyclonal; 1:8000; Dako), smooth muscle actin (1A4, 1:4000; Coulter Immunotech, Westbrook, ME, USA), desmin (D33, 1:1000; Dako), HMB-45 (prediluted; Biogenex, San Ramon, CA, USA), and Melan A (A103, 1:20; Biogenex). Appropriate positive and negative controls were performed. The cases were scored on a semiquantitative scale: $(-)=$ no staining, focal $=1-25 \%$ positive cells, variable $=26-50 \%$ positive cells, diffuse $=51-$ $100 \%$ positive cells. FISH for MDM2 gene was performed in 11 cases. All hybridization studies were performed according to a previously published protocol using a probe spanning the MDM2 gene especially developed for paraffin-embedded tissue thin sections $(4 \mu \mathrm{m}) .{ }^{10}$

\section{Results}

A total of 59 consecutive resection specimens for renal cell carcinoma were reviewed. Ten (17\%) invaded through the renal capsule into the perinephric adipose tissue. Of these cases, three (30\%) contained the aforementioned atypical cells. In contrast, 9 of $49(18 \%)$ cases without extrarenal invasion contained atypical spindle cells. Of the cases with atypical spindle cells, three (25\%) were associated with extrarenal involvement. Including the 2 cases from our consultation files, atypical spindle cells were seen in all 14 cases. Morphologically, the atypical stromal cells were distributed in a haphazard manner through the perinephric adipose tissue and embedded in a variably collagenous and myxoid stroma, occasionally within wellformed fibrous septa (Figure 1). The spindle cells were characterized by enlarged, hyperchromatic nuclei with variable amounts of amphophilic cytoplasm (Figure 1). Some of the spindle cells were plumper and more epithelioid and the nuclei were often eccentric. Scattered 'floret-type' multinucleate cells were present in three cases (Figure 1). Mitotic activity was not identified. Necrosis was not seen, nor were there any lipoblasts in the adipose tissue adjacent to the kidneys. No significant inflammation was associated, but scattered mast cells were occasionally present in the collagenous stroma. The atypical spindle cells exhibited focal $(<25 \%)$ to variable positivity (26-50\%) for smooth muscle actin and desmin in 3 of the 14 cases each (Figure 2). Cytokeratin AE1/AE3, cytokeratin Cam 5.2, cytokeratin 7 , epithelial membrane antigen, S-100, HMB-45, and Melan A were negative in all cases. No MDM2 amplification was identified by FISH in the atypical 


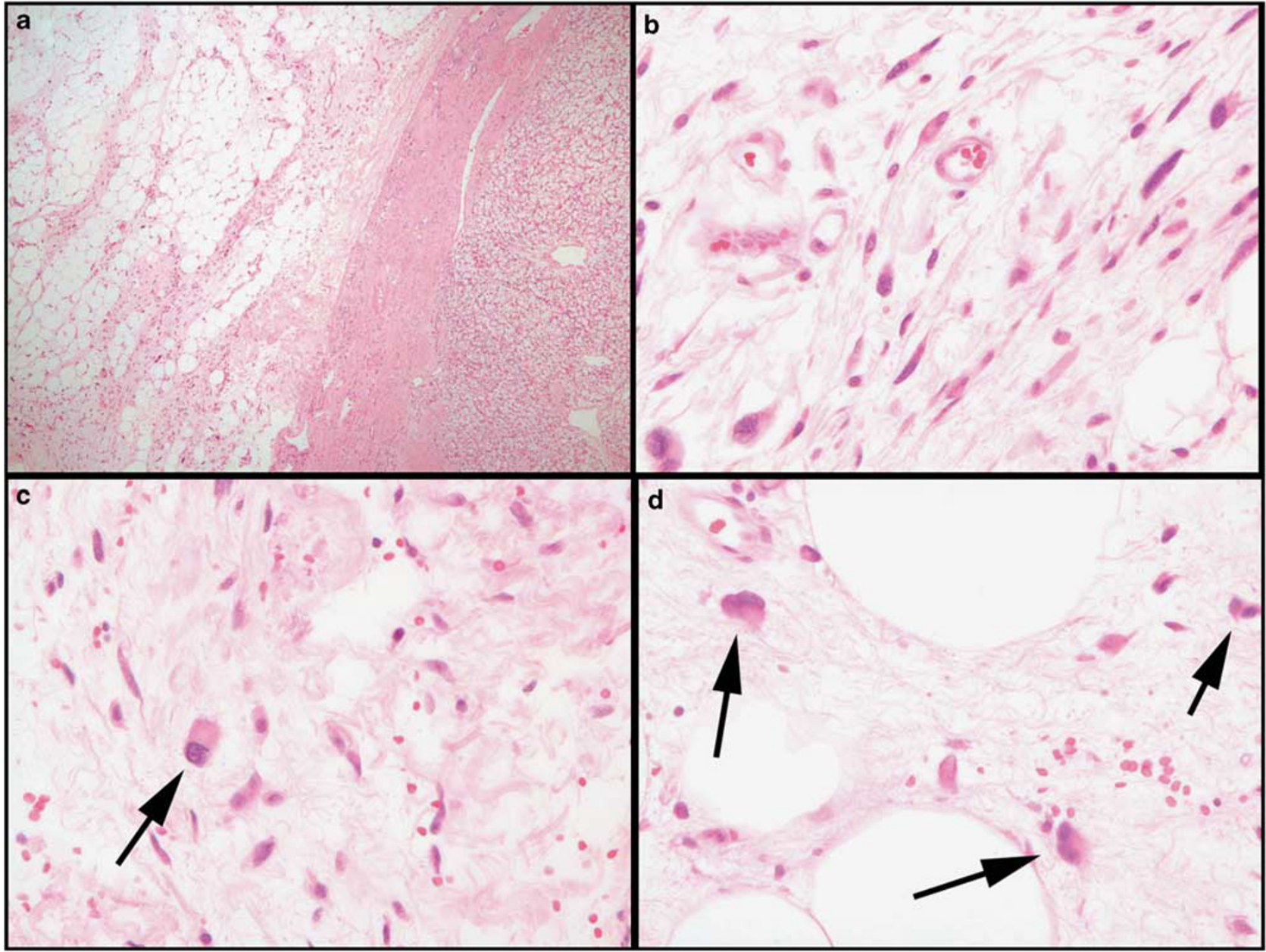

Figure 1 Features of atypical spindle-cell proliferations. (a) Fibrous septa ramify through the fat (left) adjacent to renal cell carcinoma (right). (b) High-power view of atypical spindle cells admixed with fibromyxoid stroma. Note the lesional cells have variably sized hyperchromatic nuclei. (c) Some of the cells are more epithelioid (arrow) with abundant eosinophilic to amphophilic cytoplasm and eccentrically placed nuclei. (d) 'Floret-type' multinucleate cells (arrows) were identified in four cases.

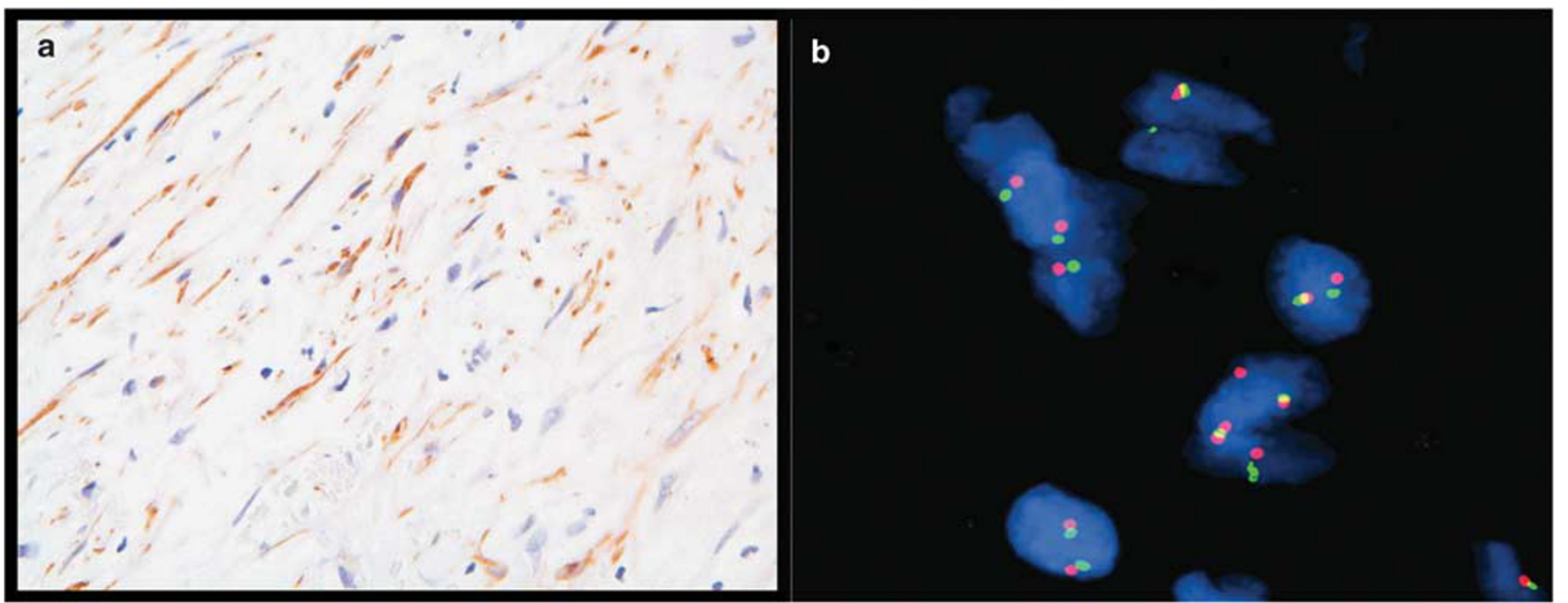

Figure 2 (a) Immunoreactivity for desmin was seen in the atypical spindle cells in three cases. (b) None of 11 cases that were tested showed amplification of the MDM2 gene region. The FITC-green-labeled probe is to the centromere of chromosome 12 and the red-rhodamine-labeled probe is to the MDM2 gene region. 
spindle cells in the 11 cases that were examined (Figure 2).

\section{Discussion}

Atypical stromal cells are not uncommonly seen in the perinephric soft tissue adjacent to the kidney in cases of renal cell carcinoma as we have described. Although not the subject of this paper, we have even seen such proliferations in two nephrectomy specimens resected for oncotyoma (DN and JM, unpublished data). Upon encountering atypical stromal cells in the perinephric tissue, several diagnostic possibilities were explored. A concurrent welldifferentiated liposarcoma was considered. Given the propensity for well-differentiated liposarcoma to arise in the retroperitoneum, one might expect that rarely, synchronous well-differentiated liposarcoma and renal cell carcinoma might occur. A review of the English language literature reveals only two cases of a concurrent retroperitoneal liposarcoma associated with renal cell carcinoma. ${ }^{11,12}$ Other sarcomas have been reported to coexist with renal cell carcinomas, but only rarely. ${ }^{13}$ Although the atypical spindle cells present in the perinephric adipose tissue were very similar to those seen in well-differentiated liposarcoma, the absence of a mass lesion made the diagnosis of well-differentiated liposarcoma unlikely. The lack of amplification of MDM2 as shown by FISH further excluded the diagnosis of well-differentiated liposarcoma. ${ }^{14,15}$

In addition, we wondered about the diagnosis of dedifferentiated liposarcoma. Dedifferentiated liposarcoma is characterized by the presence of well-differentiated liposarcoma juxtaposed with a non-lipogenic sarcoma. ${ }^{5,6}$ However, the atypical spindle cells described in this report were always admixed with adipocytes. Although this pattern can occur in dedifferentiated liposarcoma, the spindle cells lacked mitotic activity and necrosis. Furthermore, as with well-differentiated liposarcoma the absence of amplification of MDM2 argues against the diagnosis of dedifferentiated liposarcoma.

We also considered the possibility that the atypical spindle cells might represent a low-grade form of metaplastic renal cell carcinoma. Metaplastic renal cell carcinomas have been shown to contain components resembling fibrosarcoma, malignant fibrous histiocytoma (pleomorphic sarcoma not otherwise specified), and on occasion heterologous elements consisting of rhabdomyosarcoma, osteosarcoma, and chondrosarcoma. ${ }^{2-4}$ Liposarcomatous differentiation has not been described in metaplastic renal cell carcinoma, but this possibility was entertained, given the potential for metaplastic carcinomas to undergo divergent differentiation. However, the spindle cells we observed did not contain mitotic activity or necrosis, two features that are typically seen in metaplastic carcinoma. The lack of immunoreactivity for various keratins
(AE1/AE3, cytokeratin 7, and Cam 5.2) as well as epithelial membrane antigen further argued against the possibility of metaplastic renal cell carcinoma.

The possibility of fat-predominant angiomyolipoma was also considered. Synchronous occurrences of renal neoplasms and angiomyolipoma have been reported. ${ }^{16-18}$ However, no characteristic thickwalled vessels or perivascular arrangement of the atypical stromal cells was found. Furthermore, the atypical cells were negative for HMB-45 and Melan A, essentially excluding this possibility.

The last possibility, which we favored, was that the atypical spindle cells were not neoplastic, but were a bizarre pseudosarcomatous reactive proliferation. Focal to variable positivity for smooth muscle actin and desmin seen in some cases was consistent with pseudosarcomatous fibroblastic/myofibroblastic proliferations, which are within the spectrum of reactive atypia in fibroblasts/myofibroblasts that have been described. In some lesions, such as ischemic fasciitis, the atypia is degenerative, and can mimic a malignancy. ${ }^{19,20}$ Similar stromal atypia has been noted in the bladder, ${ }^{21,22}$ breast, ${ }^{23}$ nasal polyps, ${ }^{24}$ polyps and ulcers of the gastrointestinal tract, ${ }^{25}$ as well as fibroepithelial polyps present in the gynecological tract. ${ }^{26}$

In summary, we believe that the atypical stromal cells in this study represent another example of the bizarre cytological atypia fibroblasts/myofibroblasts may assume. Awareness of this phenomenon in the retroperitoneum, particularly in the setting of renal cell carcinoma, is important to avoid the potential pitfall of rendering the misdiagnosis of liposarcoma.

\section{Disclosure/Conflict of interest}

The authors declare no conflict of interest.

\section{References}

1 Eble JH, Sauter G, Epstein JI, et al. (eds). World Health Organization Classification of Tumours. Pathology \& Genetics of Tumours of the Urinary System and Male Genital Organs. IARC Press: Lyon, France, 2004.

2 Ro J, Ayala A, Sella A, et al. Sarcomatoid renal cell carcinoma: clinicopathology study of 42 cases. Cancer 1987;59:516-526.

3 de Peralta-Venturina M, Moch H, Amin M, et al. Sarcomatoid differentiation in renal cell carcinoma: a study of 101 cases. Am J Surg Pathol 2001;25:275-284.

4 Li L, Teichberg A, Steckel J, et al. Sarcomatoid renal cell carcinoma with divergent sarcomatoid growth patterns: a case report and review of the literature. Arch Pathol Lab Med 2005;129:1057-1060.

5 Fletcher CDM. Diagnostic Histopathology of Tumors, 2nd edn. Churchill Livingstone: New York, 2000, pp 1480-1483.

6 Weiss SW, Goldblum JR. Liposarcoma Enzinger and Weiss's Soft Tissue Tumors, 5th edn. Mosby: St Louis, 2008, pp 477-516. 
7 Pedeutour F, Suijkerbuijk RF, Forus A, et al. Complex composition and co-amplification of SAS and MDM2 in ring and giant rod marker chromosomes in welldifferentiated liposarcoma. Genes Chromosomes Cancer 1994;10:85-94.

8 Pedetour F, Forus A, Coindre JM, et al. Structure of the supernumerary ring and giant rod chromosomes in adipose tissue tumors. Genes Chromosomes Cancer 1999;24:30-41.

9 Sandberg AA, Bridge JA. Updates on the cytogenetics and molecular genetics of bone and soft tissue tumors: liposarcoma. Cancer Genet Cytogenet 2004; 155:1-24.

10 Trahan S, Erickson-Johnson MR, Rodriguez F, et al. Formation of the 12q14-q15 amplicon precedes the development of a well-differentiated liposarcoma arising from a nonchondroid pulmonary hamartoma. Am J Surg Pathol 2006;30:1326-1329.

11 Lewis DJ, Moul JW, Williams SC, et al. Perirenal liposarcoma containing extramedullary hematopoiesis associated with renal cell carcinoma. Urology 1994;43: 106-109.

12 Williamson JM, Konig TC, Canelo R. Incidental finding of renal cell carcinoma in recurrent retroperitoneal liposarcoma. Ann R Col Surg Engl 2008;90:W4-W5.

13 Singh EO, Barrett DM, Adams VI. Synchronously occurring malignant fibrous histiocytoma of the kidney with contralateral renal cell carcinoma. J Urol 1982;128:586-588.

14 Binh MB, Sastre-Garau X, Guillou L, et al. MDM2 and CDK4 immunostainings are useful adjuncts in diagnosing well-differentiated and dedifferentiated liposarcoma subtypes: a comparative analysis of 559 soft tissue neoplasms with genetic data. Am J Surg Pathol 2005;29:1340-1347.

15 Sirvent N, Coindre JM, Maire G, et al. Detection of MDM2-CDK4 amplification by fluorescence in-situ hybridization in 200 paraffin-embedded tumor samples: utility in diagnosing adipocytic lesions and comparison with immunohistochemistry and real-time PCR. Am J Surg Pathol 2007;31:1476-1489.

16 Csanaky G, Szereday Z, Magyarlaki T, et al. Renal angiomyolipoma: a report of three cases with regional lymph node involvement and/or with renal cell carcinoma. Tumori 1995;81:469-474.

17 Jimenez RE, Eble JN, Reuter VE, et al. Concurrent angiomyolipoma and renal cell neoplasia: a study of 36 cases. Mod Pathol 2001;14:157-163.

18 Jun SY, Cho KJ, Kim CS, et al. Triple synchronous neoplasms in one kidney: report of a case and review of the literature. Ann Diagn Pathol 2003;7:374-380.

19 Liegl B, Fletcher CD. Ischemic fasciitis: analysis of 44 cases indicating an inconsistent association with immobility or debilitation. Am J Surg Pathol 2008; 32:1546-1552.

20 Perosio PM, Weiss SW. Ischemic fasciitis: a juxtaskeletal fibroblastic proliferation with a predilection for elderly patients. Mod Pathol 1993;6:69-72.

21 Young RH. Pseudosarcomatous lesions of the urinary bladder, prostate gland, and urethra. Arch Pathol Lab Med 1987;111:354-358.

22 Young RH. Fibroepithelial polyp of the bladder with atypical stromal cells. Arch Pathol Lab Med 1986; 110:241-242.

23 Rosen PP. Multinucleated mammary stromal giant cells. A benign lesion that simulates invasive carcinoma. Cancer 1979;44:1305-1308.

24 Compagno J, Hyams VJ, Lepore ML. Nasal polyposis with stromal atypia. Review and follow-up study of 14 cases. Arch Pathol Lab Med 1976;100:224-226.

25 Shekitka KM, Helwig EB. Deceptive bizarre stromal cells in polyps and ulcers of the gastrointestinal tract. Cancer 1991;67:2111-2117.

26 Nucci MR, Young RH, Fletcher CDM. Cellular pseudosarcomatous fibroepithelial stromal polyps of the lower female genital tract: an underrecognized lesion often misdiagnosed as sarcoma. Am J Surg Pathol 2000;24:231-240. 